\begin{tabular}{|c|c|}
\hline Title & Diffusion-controlled peritectic reaction process in carbon steel analyzed by quantitative phase field simulation \\
\hline Author(s) & Ohno, Munekazu; Matsuura, Kiyotaka \\
\hline Citation & $\begin{array}{l}\text { A cta Materialia, 58(18), 6134-6141 } \\
\text { https://doi.org/10.1016/.actamat.2010.07.031 }\end{array}$ \\
\hline Issue Date & $2010-10$ \\
\hline Doc URL & http:/hdl.handle.net/2115/44156 \\
\hline Type & article (author version) \\
\hline File Information & A M58-18_61346141.pdf \\
\hline
\end{tabular}

Instructions for use 


\title{
Diffusion-controlled peritectic reaction process in carbon steel analyzed by quantitative phase-field simulation
}

\author{
Munekazu Ohno and Kiyotaka Matsuura
}

Division of Materials Science and Engineering, Faculty of Engineering, Hokkaido University, Kita 13 Nishi 8, Kita-ku, Sapporo, Hokkaido, 060-8628, Japan

\begin{abstract}
The peritectic reaction process in carbon steel, $\mathrm{L}+\delta \rightarrow \gamma$, is analyzed by a quantitative phase-field simulation. The calculated moving velocities of $\gamma$-L and $\gamma$ - $\delta$ planar interfaces in the isothermal peritectic transformation precisely agree with the corresponding experimental data, which strongly supports the accuracy of the present simulation. The diffusion-controlled peritectic reaction rate or the growing velocity of $\gamma$ phase along $\delta$-L interface obtained by the present simulation is fairly consistent with the experimentally measured value. This fact indicates that the recent experimental findings can be explained by the diffusion-controlled mechanism. This is in marked contrast to the claims made on the basis of the experimental data and an analytical model that the peritectic reaction is not controlled by the diffusion of carbon.
\end{abstract}

Keywords: Phase-field models; Peritectic solidification; Simulation; Steels 


\section{Introduction}

The peritectic reaction involves the motion of triple junction between liquid and two solid phases [1]. The rate-controlling process of the peritectic reaction observed in many alloy systems has been considered to be the diffusion of solute atom in the liquid phase. However, recent in-situ observations by means of a confocal scanning laser microscope (CSLM)[2,3] demonstrated that the peritectic reaction rate in carbon steels, viz., the growth rate of austenite $(\gamma)$ along ferrite $(\delta) /$ liquid(L) interface is too fast to be consistent with the diffusion-controlled reaction rate predicted by a theoretical model [4]. Therefore, it was concluded in these experimental studies that the peritectic reaction in the carbon steel is not controlled by the diffusion of carbon [2,3].

The aforementioned theoretical model was originally developed by Bosze and Trivedi to describe the growth process of a single phase in a matrix [4]. Figure 1 shows the phase diagram in Fe-C binary system. The liquidus and solidus lines for $\delta$ phase are extrapolated to a holding temperature (horizontal dotted line) below the peritectic temperature, $1768 \mathrm{~K} . c_{i, e}^{j}$ represents the carbon concentration in $i$ phase which is in equilibrium with $j$ phase. It is noticed that $c_{L, e}^{\gamma}$ is higher than $c_{L, e}^{\delta}$ below the peritectic temperature. The concentration in the liquid phase near the triple junction is thereby increased by the rejection of solute atom from the growing $\gamma$ phase in the peritectic reaction. It is then supposed that the melting of $\delta$ phase may occur in the vicinity of the front edge of growing $\gamma$ phase in order to reduce the increased concentration near the $\delta$ - $\mathrm{L}$ interface [5]. This process is characterized by 
the geometrical shape schematically shown in Fig. 2. It is seen that the front edge of the growing $\gamma$ phase corresponds not to the triple junction but to the $\gamma$-L interface. The motion of the front edge of $\gamma$ phase accordingly proceeds only by the $\gamma$ solidification. Assuming this process, Fredriksson and Nylén applied the Bosze and Trivedi’s theoretical model to the analysis on the peritectic reaction [5]. In the present paper, this model is called FN model. It should be pointed out that the FN model ignores the effect of solid diffusion on the peritectic reaction. Furthermore, the FN model does not explicitly deal with the motion of the triple junction and, instead, it describes only the $\gamma$ solidification. However, these assumptions have not been verified for the Fe-C system. In particular, it should not be safe to disregard the effect of the solid diffusions in Fe-C system. In view of this fact, we consider that there is no reliable value available for the diffusion-controlled peritectic reaction rate. Hence, the recent conclusions claiming diffusionless mechanisms for the peritectic reaction in the Fe-C system $[2,3]$ should be subjected to a reexamination based on a more reliable theory or numerical simulation.

The phase-field model has been developed as a powerful method to describe a variety of microstructural evolution processes. This model has been applied to the analysis on the peritectic reaction and transformation in carbon steels [6-11]. However, as discussed in the recent papers [12-14], the conventional models involve several anomalous interfacial effects. Accordingly, the outcome of the conventional model largely depends on the interface thickness assumed in the simulation and one cannot obtain the quantitatively accurate result. 
This problem was resolved in the quantitative phase-field model [13-17]. However, the available quantitative phase-field model for the two-phase solidification is applicable only to the system without the solid diffusion and with equal interfacial energies for all two-phase interfaces. In the Fe-C system, however, the solid diffusion of carbon is not negligible as will be demonstrated later and the interfacial energy is different for the different interfaces.

Recently, we developed a quantitative phase-field model for the two-phase solidification in a binary alloy system with arbitrary values of the solid diffusivities and interfacial energies [12]. The computation for the peritectic reaction process demonstrated the excellent convergence behavior of the outcome with respect to the interface thickness. Therefore, this model enables us to perform quantitatively accurate simulation for the peritectic reaction in the Fe-C system which is our main concern here.

In this study, the quantitative phase-field model developed in Ref. [12] is applied to the computation for the diffusion-controlled peritectic reaction rate in the Fe-C system. The rate-controlling process of the peritectic reaction is discussed based on the comparison between the calculated results and the reported experimental data [2,3]. The present simulation shows that the geometrical shape shown in Fig. 2 does not appear and the peritectic reaction does not proceed only by the $\gamma$ solidification. Hence, the FN model is not applicable to the Fe-C system. One will see that the diffusion-controlled reaction rate obtained by the present simulation is fairly consistent with the experimental data [2,3]. 


\section{Quantitative phase-field model}

In our recent study [12], the quantitative phase-field model was developed for the two-phase solidification in a binary alloy system with arbitrary values of the solid diffusivities and interfacial energies. The computation for the peritectic reaction in the Fe-C system demonstrated the excellent convergence behavior of the outcome with respect to the interface thickness. Therefore, this model allows us to carry out the quantitatively accurate simulation for the peritectic reaction process, as long as the reliable values of input parameters are available. Since the main focus of our previous study [12] was placed on the development of the quantitative phase-field model and its computational performance, the details of the reaction were not investigated. In this study, we perform the detailed analysis on the peritectic reaction in the $\mathrm{Fe}-\mathrm{C}$ system. We briefly summarize the essential parts of the quantitative phase-field model developed in Ref. [12].

In the phase-field model, the existence of $i$ phase is characterized by the phase field variable, $p_{i}$, which continuously varies from 1 to 0 . The subscript $i$ specifies the type of phases such as $i=\mathrm{L}, \delta$ and $\gamma \cdot p_{i}$ represents the probability of finding $i$ phase. These phase fields satisfy the normalization condition as given by $\Sigma_{i} p_{i}=1$. The summation is carried out over $i=\mathrm{L}, \delta$ and $\gamma$. For convenience, we use the notation $p_{j}$ and $p_{k}$ to distinguish the different phases. The solute concentration, $c$, is described by the mixture rule, $c=\Sigma c_{i} p_{i}$ where $c_{i}$ is the concentration in $i$ phase. Then, the time evolution of the phase field, $p_{i}$, is described by the following equation [12], 


$$
\begin{aligned}
\frac{1}{M\left(\left\{p_{i}\right\}\right)} \frac{\partial p_{i}}{\partial t}=\sum_{k} & m_{k}\left(\frac{1}{2} \varepsilon^{2} \nabla^{2} p_{k}-2 \omega\left(\left\{p_{i}\right\}\right) p_{k}\left(1-p_{k}\right)\left(1-2 p_{k}\right)\right. \\
& \left.-\frac{\partial \omega\left(\left\{p_{i}\right\}\right)}{\partial p_{k}}\left(\sum_{j} p_{j}{ }^{2}\left(1-p_{j}\right)^{2}\right)-\sum_{j} \frac{\partial g_{j}}{\partial p_{k}}\left(f_{j}-\mu_{c} c_{j}\right)\right) .
\end{aligned}
$$

$M\left(\left\{p_{i}\right\}\right)$ is the mobility given as $M\left(\left\{p_{i}\right\}\right)=2\left(\Sigma^{*} M_{i j} p_{i} p_{j}\right)\left(\Sigma^{*} p_{i} p_{j}\right)^{-1}$ where $\Sigma^{*}$ denotes the summation over $(i, j)=(\delta, L),(\gamma, L),(\gamma, \delta)$ and $M_{i j}$ is the mobility for $i-j$ interface as explicitly described later. $m_{k}$ is a constant given as $m_{k}=-2 / 3$ for $k=i$ and $m_{k}=1 / 3$ for $k \neq i$. $\varepsilon$ is the gradient energy coefficient. $\omega\left(\left\{p_{i}\right\}\right)$ determines the contribution of the double well potentials and it is written as,

$$
\omega\left(\left\{p_{i}\right\}\right)=\left(2 \sum^{*} p_{i}^{2} p_{j}^{2}\right)^{-1}\left(\sum^{*} \omega_{i j} p_{i}^{2} p_{j}^{2}+\omega_{t r i}\left(p_{i} p_{j} p_{k}\right)^{n}\right),
$$

where the exponent $n$ is larger than 1 and $\omega_{i j}$ indicates the potential height for $i-j$ interface. $\omega_{t r i}$ controls the potential height for the mixture state of three phases. In eq.(1), $g_{j}$ is the interpolating function defined as $g_{j}\left(\left\{p_{j}\right\}\right)=\left(p_{j}^{2} / 4\right)\left\{15\left(1-p_{j}\right)\left[1+p_{j}-\left(p_{k}-p_{i}\right)^{2}\right]+p_{j}\left(9 p_{j}{ }^{2}-5\right)\right\}[14] . f_{j}$ is the free energy density for the bulk $j$ phase and $\mu_{c}$ is the chemical potential. In the derivation of eq.(1), the condition for the equal chemical potential, $\mu_{c}=\partial f_{i} / \partial c_{i}=\partial f_{j} / \partial c_{j}=\partial f_{k} / \partial c_{k}$, is introduced. The last term in the parenthesis of eq.(1) represents the thermodynamic driving force. This term can be approximated for the dilute solid solution as $f_{j}-\mu_{c} C_{j} \approx V_{m}{ }^{-1} R T\left(-C_{k, e}{ }^{j}+c_{j, e}{ }^{k}-C_{j}\right)$ where $v_{m}$ is the molar volume, $R$ is the gas constant and $k$ phase corresponds to a reference phase which was chosen to be the liquid phase in our simulation.

The time evolution of concentration field, $c$, is described by the following diffusion equation [12], 


$$
\frac{\partial c}{\partial t}=\nabla\left(\sum_{i} D_{i} p_{i} \nabla c_{i}+2 \sum^{*}\left(\mathbf{n}_{i} \cdot \mathbf{n}_{j}\right) a_{i j} \frac{\varepsilon}{\sqrt{\omega_{i j}}}\left(c_{j}-c_{i}\right) \frac{\partial p_{i}}{\partial t} \mathbf{n}_{i}\right),
$$

where $D_{i}$ is the diffusion coefficient of the solute atom in $i$ phase, $\mathbf{n}_{i}$ is given by $\mathbf{n}_{i}=-\nabla p_{i} /\left|\nabla p_{i}\right| \cdot a_{i j}$ is written as,

$$
a_{i j}=\frac{1}{2 \sqrt{2}}\left(D_{j}-k_{i j} D_{i}\right)\left[1-\frac{1}{2}\left(1-k_{i j} \frac{D_{i}}{D_{j}}\right) \chi_{i j}\right]
$$

where $k_{i j}$ is the partition coefficient defined as $k_{i j}=c_{i} / c_{j}$ and $\chi_{i j}$ is a parameter controlling the convergence behavior of the output with respect to the interface thickness. The second term in the parenthesis of eq.(3) indicates the antitrapping current term which is introduced to eliminate the anomalous interface effects especially originating from the abnormal solute trapping[18-20].

Within the present model, the $i-j$ interfacial energy, $\sigma_{i j}$, is described by $\sigma_{i j}=\varepsilon\left(\omega_{i j}\right)^{1 / 2} /\left(3 \cdot 2^{1 / 2}\right)$. The thickness of $i-j$ interface, $W_{i j}$, is given by $W_{i j}=\varepsilon / \omega_{i j}{ }^{1 / 2}$. Within the thin-interface limit, also, the mobility for $i-j$ interface, $M_{i j}$, is calculated as follows,

$$
M_{i j}=\left(\frac{15}{4} \frac{a_{2} \varepsilon^{2} R T_{m, i j}}{\omega_{i j} D_{j} v_{m}}\left(1-k_{i j}\right)\left(c_{j, e}^{i}-c_{i, e}^{j}\right)\left[1-\frac{1}{2}\left(1-k_{i j} \frac{D_{i}}{D_{j}}\right) \chi_{i j}\right]\right),
$$

where $a_{2}=0.6276 \cdots$ and $T_{m, i j}$ is the transition temperature between $i$ and $j$ phases at $c=0$.

In the present model, the solid diffusion is explicitly taken into account, which is of critical importance in analyzing the peritectic reaction process, and the inequality of interfacial energies can be dealt with. It should be noticed that the present model is free from the problem associated with the unexpected formation of extra phase in the interface as discussed 
in Ref. [12]. The formation of the extra phase can be successfully suppressed by introduction of the additional potential, the term multiplied by $\omega_{t r i}$ in eq.(2). The contribution of this additional potential is controlled by the values of $n$ and $\omega_{\text {tri }}$ that turn out to be the parameters controlling the convergence behavior of the outcome with respect to the interface thickness. The most important fact is that the present model is exactly reduced to the available thin-interface limit model for the single phase solidification [20].

We solved the time evolution equations (1) and (3) based on the standard second-order finite difference method. We employed the computational grid size of $\Delta x=1.0 \times 10^{-8} \mathrm{~m}$ in all the simulations described in the next section. The time step, $\Delta t$, was varied from $\Delta t=5 \times 10^{-11}$ to $\Delta t=1 \times 10^{-10} \mathrm{~s}$, depending on the holding temperature. The interface thicknesses were set to be $W_{\gamma \delta}=2 \cdot \Delta x, W_{\delta \mathrm{L}}=\sigma_{\gamma \delta} W_{\gamma \delta} / \sigma_{\delta \mathrm{L}}$ and $W_{\gamma \mathrm{L}}=\sigma_{\gamma \delta} W_{\gamma \delta} / \sigma_{\gamma \mathrm{L}}$. We used $\sigma_{\delta \mathrm{L}}=0.204$ [21], $\sigma_{\gamma \mathrm{L}}=0.319$ [22] and $\sigma_{\gamma \delta}=0.370 \mathrm{~J} \cdot \mathrm{m}^{-2}$ [23].The diffusion coefficient was given in the form, $D_{i}=a_{i} \cdot \exp \left(-Q_{i} /(R T)\right) \mathrm{m}^{2} / \mathrm{s}$ and we used $a_{\mathrm{L}}=5.2 \times 10^{-7}, Q_{\mathrm{L}}=5.0 \times 10^{4}, a_{\delta}=1.27 \times 10^{-6}, Q_{\delta}=8.3 \times 10^{4}$, and $a_{\gamma}=7.61 \times 10^{-6}, Q_{\gamma}=13.7 \times 10^{4}[24]$. For the parameters associated with the thermodynamic driving force, we employed the following values estimated from the thermodynamic assessment of Ref. [25], $k_{\delta \mathrm{L}}=0.179, k_{\gamma \mathrm{L}}=0.334, k_{\gamma \delta}=k_{\gamma \mathrm{L}} / k_{\delta \mathrm{L}}, T_{m, \delta \mathrm{L}}=1881 \mathrm{~K}, T_{m, \gamma \mathrm{L}}=1801 \mathrm{~K}$, $T_{m, \gamma \delta}=1399 \mathrm{~K}, \quad c_{L, e}^{\delta}(T)=\left(T_{m, \delta \mathrm{L}}-T\right) / 1828, \quad c_{L, e}^{\gamma}(T)=\left(T_{m, \gamma \mathrm{L}}-T\right) / 1399$. As for the parameters controlling the convergence behavior of the output, we employed $\chi_{i j}=0.0, n=1.4$ and $\omega_{t r i}=\omega_{\delta \mathrm{L}}+\omega_{\gamma \mathrm{L}}+\omega_{\gamma \delta}$, since the simulations with these values yield the well converged results as demonstrated in Ref. [12]. It is noted that the dependence of physical parameters such as the 
diffusion coefficient $D_{i}$ and interfacial energy $\sigma_{i j}$ on the carbon concentration is not taken into consideration.

\section{Results and discussion}

\subsection{Motion of planar interfaces in the peritectic transformation}

In order to check the accuracy of the present quantitative simulation, we first

calculated the moving velocities of $\gamma-\delta$ and $\gamma$-L planar interfaces in the isothermal peritectic transformation. In the experimental studies in Refs. [26,27], the moving velocity of each interface was measured by using a solid/liquid diffusion couple experiment. The diffusion couple consisted of $\delta$-iron sample (solid sample) with the carbon content of $c_{\delta, e}^{\gamma}$ and melted high carbon steel sample (liquid sample) with $c_{L, e}^{\gamma}$. The purity of the solid and liquid samples was $\mathrm{Si}<0.01, \mathrm{Mn}<0.01, \mathrm{P}<0.002, \mathrm{~S}<0.002$ and $\mathrm{Al}<0.01$ mass $\%$. These samples were held in contact with each other at a holding temperature. Then, the $\gamma$ phase formed between the solid and liquid samples. The thickness of the $\gamma$ phase increased with the holding time. In these experiments, the $\gamma$-L and $\gamma-\delta$ interfaces exhibited almost planar shape. Therefore, this situation can be regarded as the one-dimensional moving boundary problem. The moving distances of the $\gamma$-L and $\gamma-\delta$ planar interfaces were measured by the microstructural observation of the quenched samples. It was found that the time dependence of the moving distance for $i-j$ interface, $\Delta x_{i j}$, follows the relation, $\Delta x_{i j}=A_{i j} t^{1 / 2}$, with a constant $A_{i j}$, which is called the parabolic rate constant in this report. In order to check the accuracy of the present simulation, 
we performed the following simulation and compare the result with these experimental data $[26,27]$.

We employed the one-dimensional system which initially consists of the liquid phase with $c_{L, e}^{\gamma}$ and $\delta$ phase with $c_{\delta, e}^{\gamma}$. Also, the $\gamma$ phase with $c_{\gamma, e}^{\delta}$ exists between the liquid and $\delta$ phases. The initial thickness of the $\gamma$ phase was set to be $0.7 \mu \mathrm{m}$. The zero flux boundary conditions were employed for $p_{i}$ and $c$ at both ends of the system. In order to avoid the influence of the system size on the interface motion, we used a relatively large system size of $8 \mu \mathrm{m}$. After the concentration profile was relaxed for a certain time period during the isothermal annealing operation, the moving distance of each interface from each initial position was measured. This simulation describes the motion of the planar interfaces in the isothermal transformation, of which the condition is equivalent to that in the diffusion couple experiments [26,27].

The concentration profiles calculated at $T=1750 \mathrm{~K}$ are shown in Fig. 3(a). The origin of the spatial coordinate corresponds to the initial position of $\gamma-\mathrm{L}$ interface. The $\gamma-\delta$ and $\gamma-\mathrm{L}$ interfaces move toward the left-hand and right-hand sides, respectively, increasing the thickness of $\gamma$ phase. The moving distances of the interfaces from their initial positions are plotted with respect to the holding time in Fig. 3(b). The moving velocity of the $\gamma-\delta$ interface is much higher than that of the $\gamma-\mathrm{L}$ interface at each temperature. This is quite consistent with the experimental findings [26,27]. The concentration difference at $\gamma-\delta$ interface is smaller than that at $\gamma$-L interface and, therefore, the $\gamma-\delta$ interface can migrate at a velocity higher than 
the $\gamma$-L interface. Both the velocities increase with the decrease in the temperature. This is because the difference between $c_{\gamma, e}^{L}$ and $c_{\gamma, e}^{\delta}$ becomes large at low temperature and the carbon diffusion in $\gamma$ phase accordingly becomes fast at the low temperature.

The calculated time dependence of the moving distance for each interface was well described by the relation, $\Delta x_{i j}=A_{i j} \cdot t^{1 / 2}$. The calculated parabolic rate constant, $A_{i j}$, for each interface at different temperatures is shown in Fig. 4 where the experimental data $[26,27]$ are also plotted. One can see that the calculated results are in good accordance with the experimental data over the whole temperature range. The agreement is especially excellent in the high temperature region. This agreement strongly supports the accuracy of our quantitative phase-field simulation.

It is noted that the value of interfacial energy is not relevant to the analysis on the motion of planar interface. Hence, the analysis on the planar interface thus described does not provide any information about the accuracy of the interfacial energies used in this study. Although we chose the reliable values for the interfacial energies available in the literatures [21-23], the measurement or computation of this quantity is prone to involve a relatively large uncertainty in the accuracy. This point will be discussed later in the following section.

\subsection{Peritectic reaction}

For the analysis on the isothermal peritectic reaction, we used two-dimensional system which is schematically shown in Fig. 5 [12]. We employed the zero flux boundary 
conditions for $p_{i}$ and $c$ on all the boundaries of the system. The $\delta$ and L phases initially coexist and forms the planar $\delta$-L interface. The semicircular $\gamma$ phase was put between the $\delta$ and $\mathrm{L}$ phases and then, the $\gamma$ phase grows along the $\delta$-L interface ( $y$ direction). We calculated the velocity of the front edge of growing $\gamma$ phase, viz., the peritectic reaction rate at a given degree of undercooling from the peritectic temperature, $\Delta T$. All the results shown here correspond to those in the steady state. We considered that the system reaches the steady state when the peritectic reaction rate takes almost a constant value with respect to the time. The values of $W_{x}$ and $W_{y}$ were chosen to be large enough to avoid the influence of system size on the reaction rate [12]. For example, we chose $W_{x} \geq 6 \mu \mathrm{m}$ and $W_{y} \geq 3 \mu \mathrm{m}$ for $\Delta T=5 \mathrm{~K}$ and $W_{x} \geq 6 \mu \mathrm{m}$ and $W_{y} \geq 3 \mu \mathrm{m}$ for $\Delta T=10 \mathrm{~K}$. The values of $c_{\gamma}^{0}$ and $d_{i}$ are irrelevant to the steady state velocity of our interest [12]. We set $c_{\gamma}^{0}=c_{\gamma, e}^{L}$ and $d_{i}=40 \times 10^{-8} \mathrm{~m}$ in all the simulations.

\subsubsection{Melting of $\delta$ phase}

As described in the introduction, the diffusion-controlled peritectic reaction rate, $V_{p}$, has been estimated based on the FN model [5], which is expressed as,

$$
V_{p}=\frac{9}{8 \pi} \frac{D_{L}}{\rho}\left[\frac{2 \pi \Omega}{2 \pi-4 \Omega-\Omega^{2}}\right]^{2}
$$

where $\rho$ is the thickness of plate-like $\gamma$ phase, $\Omega$ is given as $\Omega=\left(c_{L, e}^{\gamma}-c_{L, e}^{\delta}\right) /\left(c_{L, e}^{\gamma}-c_{\gamma, e}^{L}\right)$. It should be stressed that the FN model does not take the solid diffusions into account. As demonstrated in the previous section, however, the moving velocity of $\gamma-\delta$ interface is quite high compared to that of $\gamma-\mathrm{L}$ interface. This result clearly indicates that the solid diffusions 
cannot be neglected in the peritectic reaction in the Fe-C system. Moreover, the FN model is based on the assumption that the peritectic reaction proceeds only by the $\gamma$ solidification as shown in Fig. 2. The melting of $\delta$ phase near triple junction is assumed to take place due to the concentration difference between $c_{L, e}^{\delta}$ and $c_{L, e}^{\gamma}$. The geometrical shape shown in Fig. 2 is the essential assumption in the FN model.

The calculated shape of interfaces in the vicinity of the triple junction is shown in Fig. 6. In each figure, the level 0.5 contour lines of the phase fields are demonstrated. The origin of $y$ axis corresponds to the position of the triple junction defined by $p_{\mathrm{L}}=p_{\delta}=p_{\gamma}=1 / 3$, while the origin of $x$ axis is the initial position of $\delta$-L interface. In the triple junction region, there exists a gap (triangle-shaped region) surrounded by the interfaces, because the level 0.5 contours of phase fields, by definition, do not meet at the triple junction defined by $p_{\mathrm{L}}=p_{\delta}=p_{\gamma}=1 / 3$. It is seen in all the figures that the $\delta$-L interface position near the triple junction deviates from the initial position $(x=0)$ toward the left-hand side. This deviation indicates that the melting of $\delta$ phase occurs in the reaction. However, the melting of $\delta$ phase only slightly takes place at the front of growing $\gamma$ phase in all the cases. The front edge of the growing $\gamma$ phase corresponds not to $\gamma$-L interface but to the triple junction. These shapes are in contrast to the shape shown in Fig. 2.

Figure 7 shows the spatial profile of concentration difference, defined as $c-c_{L, e}^{\delta}$ in the liquid phase region and $c_{\delta, e}^{L}-c$ in $\delta$ phase region. This concentration difference indicates the deviation of the concentration from $\delta$-L phase equilibrium. The concentration in the $\gamma$ 
phase is not shown here. The definitions of $x$ and $y$ axes are the same as those in Fig. 6. The concentration profiles at positive and negative $y$ values correspond to those before and after the peritectic reaction, respectively. The concentration in liquid phase is enriched ahead of growing $\gamma$ phase by $2-3 \times 10^{-4}$ at $\Delta T=5 \mathrm{~K}$ and by $3-4 \times 10^{-4}$ at $\Delta T=10 \mathrm{~K}$. This enrichment leads to the melting of $\delta$ phase as can be realized from the movement of the profile in the liquid toward the left-hand side with the decrease in $y$ value from 0.4 to $0.2 \mu \mathrm{m}$. However, the melting is not substantial at all. The results shown in Figs. 6 and 7 are in marked contrast to the assumption in the FN model. It is not safe to assume that the peritectic reaction in the Fe-C system proceeds only by the $\gamma$ solidification. Therefore, the applicability of the FN model to the Fe-C system cannot be substantiated.

The geometrical shape shown in Fig. 2 was originally proposed by Hillert [28]. He assumed that the contact angles between interfaces at the triple junction follow the thermodynamic equilibrium relation described by the Young's law. In addition, if there are no diffusions in the solids, the position of $\gamma-\delta$ interface is always located behind the triple junction. These assumptions lead to the geometrical shape shown in Fig. 2. In Fig. 6(a), it was confirmed that the calculated contact angles between the interfaces at $\Delta T=0.25 \mathrm{~K}$ obey the Young's law. When the angle between $i-j$ and $i-k$ interfaces at the triple junction is denoted by $\theta_{i}$, the Young's law describes the relation, $\theta_{i}=\cos ^{-1}\left[\left(\sigma_{j k}{ }^{2}-\sigma_{i j}{ }^{2}-\sigma_{i k}{ }^{2}\right) /\left(2 \sigma_{i j} \sigma_{i k}\right)\right]$. Then, the contact angles are calculated to be $\theta_{\mathrm{L}}=92.9^{\circ}, \theta_{\delta}=120.6^{\circ}, \theta_{\gamma}=146.6^{\circ}$ by using $\sigma_{\delta \mathrm{L}}=0.204, \sigma_{\gamma \mathrm{L}}=0.319$ and $\sigma_{\gamma \delta}=0.370 \mathrm{~J} \cdot \mathrm{m}^{-2}$. In Fig. 6, the contact angle, $\theta_{i}$, can be calculated as follows [29]. First, a 
circle with a radius $R$ is drawn. The center point of this circle is placed at the triple junction. One obtains the intersection points between this circle and each interface. Then, three lines can be drawn by connecting each intersection point and the triple junction point. One can measure $\theta_{i}$ based on these lines. The calculation for the contact angles at $\Delta T=0.25 \mathrm{~K}$ (Fig. 6(a)) with $R=2.5 W_{\delta \gamma}$ yields $\theta_{\mathrm{L}}=92.4^{\circ}, \theta_{\delta}=121.6^{\circ}, \theta_{\gamma}=146.0^{\circ}$. These values are in excellent agreement with the above-mentioned values by the Young's law. In the results for $\Delta T=5.0$ and 10 K (Figs. 6(b) and (c)), the interfaces near the triple junction appreciably deviate from flat shape. Hence, the calculated value of contact angle largely depends on the choice of $R$ and it does not allow the precise determination of the contact angle. However, the shapes of the interfaces very close to the triple junction at $\Delta T=5.0$ and $10 \mathrm{~K}$ do not significantly differ from the shape predicted by the Young's law. Therefore, it should be pointed out that the inadequacy of the Hillert's proposal in the Fe-C system mainly originates from the disregard of the solid diffusions in the reaction process.

The shape similar to Fig. 2 was actually observed in the experimental study using the CSLM [3], although a careful attention should be directed to the resolution in their observation and a further investigation seems to be required to obtain the conclusive evidence. The present simulation demonstrated that the shape in Fig. 2 is not realized only by taking the carbon diffusion into consideration. As discussed in Ref. [3], the experimentally observed shape may be attributable to the effect of latent heat released by the growing $\gamma$ phase. The latent heat released by the growing $\gamma$ phase may be partly compensated by the melting of $\delta$ 
phase, which results in the shape shown in Fig. 2. Hence, the analysis on the peritectic reaction essentially requires coupling between the solute concentration and temperature fields to be dealt with. This is however beyond the focus of the present study. Our main concern is the computation for the accurate value of the peritectic reaction rate controlled by the diffusion of carbon, which is discussed below.

\subsubsection{Peritectic reaction rate}

As discussed in the introduction, the peritectic reaction in carbon steels was investigated by the in-situ CSLM observation $[2,3]$. The peritectic reaction rate was measured to be $V_{p}=1.5-5.5 \mathrm{~mm} / \mathrm{s}$ in the range of $\Delta T=5-15 \mathrm{~K}$ in $\mathrm{Fe}-0.14$ mass\% $\mathrm{C}$ and $\mathrm{Fe}-0.42 \mathrm{mass} \% \mathrm{C}$ steels [2]. As is consistent with this measurement, the recent investigation based on the CSLM showed that the peritectic reaction rate varies from 0.4 to $12.5 \mathrm{~mm} / \mathrm{s}$ in 0.18 mass\% C steel [3]. The degree of undercooling for the data was not specified in Ref. [3] due to the experimental difficulty in the temperature measurement. On the other hand, the FN model given in eq.(6) yields $V_{p}=0.0-0.1 \mathrm{~mm} / \mathrm{s}$ in the range of $\Delta T=0-15 \mathrm{~K}[2,3]$. Hence, the experimentally measured rate was found to be much higher than the value predicted by the FN model, which led to the conclusion that the peritectic reaction is not controlled by the diffusion of carbon.

Figure 8 demonstrates the peritectic reaction rate obtained by the present simulation. The solid line with full symbols indicates the calculated results and the open symbols are the experimental data in Ref. [2]. One can see that the experimentally measured values fall within 
the calculated range of the diffusion-controlled reaction rate. Also, the calculated results are fairly consistent with the measured values of $0.4-12.5 \mathrm{~mm} / \mathrm{s}$ in Ref. [3]. The present calculation, therefore, suggests that the reported experimental findings [2,3] can be explained by the diffusion-controlled mechanism.

As already mentioned, the measured or computed value for the interfacial energy generally involves a relatively large uncertainty in the accuracy. In the present study, we employed $\sigma_{\delta \mathrm{L}}=0.204$ [21], $\sigma_{\gamma \mathrm{L}}=0.319$ [22] and $\sigma_{\gamma \delta}=0.370 \mathrm{~J} \cdot \mathrm{m}^{-2}$ [23]. It was reported that $\sigma_{\gamma \delta}$ varies from 0.37 to $0.45 \mathrm{~J} \cdot \mathrm{m}^{-2}$ depending on the sulfur content in the steel [23]. Therefore, we tested the different values of $\sigma_{\gamma \delta}$. The results are shown in Fig. 9. The degree of undercooling was set to be $\Delta T=10 \mathrm{~K}$. It should be pointed out that the simulations with $\sigma_{\gamma \delta}=0.45$ and 0.50 $\mathrm{J} \cdot \mathrm{m}^{-2}$ are not completely free from the problem associated with the formation of the extra phase in the interface [12]. A small amount of the liquid phase exists in the $\gamma-\delta$ interface region in the present numerical condition. Although this problem causes an overestimation of $V_{p}$, the error should not be substantially large. In Fig. 9(a), $V_{p}$ gradually decreases from 18 to $9.5 \mathrm{~mm} / \mathrm{s}$ as $\sigma_{\gamma \delta}$ increases from 0.18 to $0.37 \mathrm{Jm}^{-2}$. Importantly, $V_{p}$ does not change significantly in the realistic range of $\sigma_{\gamma \delta}>0.37 \mathrm{~J} \cdot \mathrm{m}^{-2}$. Hence, the change of $\sigma_{\gamma \delta}$ does not alter our conclusion that the experimentally measured reaction rate $[2,3]$ can be explained by the diffusion-controlled mechanism. The shapes of the interfaces are shown in Fig. 9(b). As $\sigma_{\gamma \delta}$ increases, the amount of the melting $\delta$ phase increases. This is consistent with the prediction by the Young's law which describes that the higher value of $\sigma_{\gamma \delta}$ results in the smaller value of 
$\theta_{\mathrm{L}}$ and the larger values of $\theta_{\delta}$ and $\theta_{\gamma}$. It is noted that when $\sigma_{\gamma \delta}$ is larger than $0.523 \mathrm{~J} \cdot \mathrm{m}^{-2}$, viz., $\sigma_{\delta \mathrm{L}}+\sigma_{\gamma \mathrm{L}}$, the $\gamma-\delta$ interface does not form in this system and the triple junction vanishes. Then, the $\gamma$ phase forms only by the peritectic transformations, viz., $\delta \rightarrow \gamma$ and $\mathrm{L} \rightarrow \gamma$ transformations. This is not the peritectic reaction observed in the experimental studies [2,3] and the analysis on this process is beyond our main concern. In Fig. 9(b), the front edge of the $\gamma$ phase corresponds to the triple junction in all the cases tested here. Therefore, the FN model cannot be validated in the Fe-C system even for the high value of $\sigma_{\gamma \delta}$.

In Fig. 8, the experimental data [2] considerably depend on the average carbon concentration. As mentioned in the section 2, we did not take into account the concentration dependence of the physical parameters. In our calculation, hence, the difference in the average carbon concentration merely results in the difference in initial volume fraction of $\delta$ phase. This change of the initial volume fraction does not cause any change in the peritectic reaction process in an infinitely large system. The concentration dependence of the physical parameters such as $D_{i}$ and $\sigma_{i j}$ may be one of the factors to explain the concentration dependence of the experimental data. Also, we focused on the process in an infinitely large system. In reality, however, the system size is finite. The experimental data in Ref. [2] should not be completely free from the size effect, since the amount of $\delta$ phase before the reaction affects heat transfer during the peritectic reaction. Moreover, the initial concentrations in $\delta$ and liquid phases were $c_{\delta, e}^{L}$ and $c_{L, e}^{\delta}$, respectively, in our simulation. This condition might not be exactly realized in the experimental study. For a further discussion, in addition, a 
careful attention may need to be paid to uncertainty in the experimental data regarding, e.g., the accuracy for the temperature measurement. Although these details remain to be addressed, we would like to stress that the results obtained by the present simulation is fairly consistent with the experimental data reported in Refs. [2, 3].

\section{Conclusions}

In this paper, the peritectic reaction process in the Fe-C system was analyzed based on the quantitative phase-field model developed in Ref. [12]. Our computation demonstrated that the melting of $\delta$ phase only slightly takes place near the triple junction. This result denies the validity of the assumption proposed by Fredriksson and Nylén that the peritectic reaction occurs only by the $\gamma$ solidification. The calculated results for the peritectic reaction rate are fairly consistent with the experimentally measured values. Therefore, the experimental findings can be explained by the diffusion-controlled mechanism. This is in marked contrast to the recent claims that the peritectic reaction in the carbon steel is not controlled by the diffusion of carbon. For a step further toward the analysis on realistic process, it is important to address the effect of the temperature field on the reaction.

Before closing, the following point should be noted regarding $\delta \rightarrow \gamma$ transformation. The CSLM observation in Ref. [2] indicated the possibility for a diffusionless transformation from $\delta$ to $\gamma$ phase in the peritectic carbon steel. Furthermore, the recent in-situ synchrotron X-ray imaging analysis demonstrated that the $\delta \rightarrow \gamma$ transformation in the peritectic carbon 
steel may occur in a diffusionless mechanism under a large undercooling [30]. These evidences are not contradiction to our discussion for the steady state behavior of the peritectic reaction. These evidences indicate that several modes may be possible in the non-equilibrium solidification of the peritectic carbon steels. The theoretical analysis on these phenomena is the important task to be attempted in the future to progress our understanding of the peritectic solidification in carbon steels.

\section{Acknowledgments}

This work is supported by $18^{\text {th }}$ ISIJ Research Promotion Grant and Grant-in-Aid for Challenging Exploratory Research 21656192 from MEXT, Japan. Also, the author M.O. acknowledges partial financial support from the Next Generation Super Computing Project, Nano-science Program, MEXT, Japan. 


\section{References}

[1] Kerr HW, Cisse J and Bolling GF, Acta Mater 1974;22;677.

[2] Shibata H, Arai Y, Suzuki M and Emi T, Metall Mater Trans B 2000; 31B; 981.

[3] Phelan D, Reid M, Dippenaar R, Mater Sci Eng A 2008; 477;226.

[4] Bosze WP and Tridedi R, Metall Trans 1974;5;511.

[5] Fredriksson Nylén HT, Met Sci 1982; 16; 283.

[6] Tiaden J, J Cryst Growth 1999; 198/199; 1275.

[7] Lee JS, Kim SG, Kim WT and Suzuki T, ISIJ Int 1999;39;730.

[8] Ode M, Kim SG, Kim WT and Suzuki T, ISIJ Int 2005; 45;147.

[9] Phelan D, Reid M, Dippenaar R, Comp Mater Sci 2005;34;282.

[10] Phelan D, Reid M, Dippenaar R, Metall Mater Trans A 2006; 37A; 985.

[11] Choudhury A, Neslter B, Telang A, Selzer M, Wendler F, Acta Mater 2010; 58; 3815.

[12] Ohno $M$ and Matsuura K, submitted to Acta Materialia.

[13] Floch R and Plapp M, Phys Rev E 2003;68;010602(R).

[14] Floch R and Plapp M, Phys Rev E 2005; 72; 011602.

[15] Plapp M, J Crys Growth 2007;303; 49.

[16] Parisi A and Plapp M, Acta Mater 2008; 56; 1348.

[17] Boussinot G, Brener EA, Temkin DE, Acta Mater 2010;58;1750.

[18] Karma A, Phys Rev Lett 2001; 87; 115701.

[19] Echebarria B, Floch R Karma A, Plapp M, Phys Rev E 2004; 70; 061604.

[20] Ohno M and Matsuura K, Phys Rev E 2009; 79;031603.

[21] Kurz W and Fisher DJ, Fundamentals of Solidification, Trans Tech Publication (Switzerland, 1989).

[22] Sun Y, Asta M, Hoyt JJ, Mendelev MI and Srolovitz DJ, Phys Rev B 2004;69;020102(R). [23] Yin H, Emi T and Shibata H, Acta Mater 1999;47;1523.

[24] Matsumiya T, Kajioka H, Mizoguchi S, Ueshima $\mathrm{Y}$ and Esaka H, Trans ISIJ 1984;24;873.

[25] Gustafson P, Scand J Metall 1985;14; 259.

[26] Matsuura K, Itoh Y and Narita T, ISIJ Int 1993;33;583.

[27] Matsuura K, Maruyama H, Itoh Y, Kudoh M and Ishii K, ISIJ Int 1995;35;183.

[28] Hillert M, Solidification and Casting of Metals, The Metals Society (London, 1979).

[29] Villanueva W, Boettinger WJ, Warren JA, Amberg G, Acta Mater 2009;57;6022.

[30] Yasuda H, Nakatsuka N, Nagira T, Yoshiya M, Sugiyama A, Uesugi K, Umetani, K, CAMP-ISIJ 2009;22;800. 


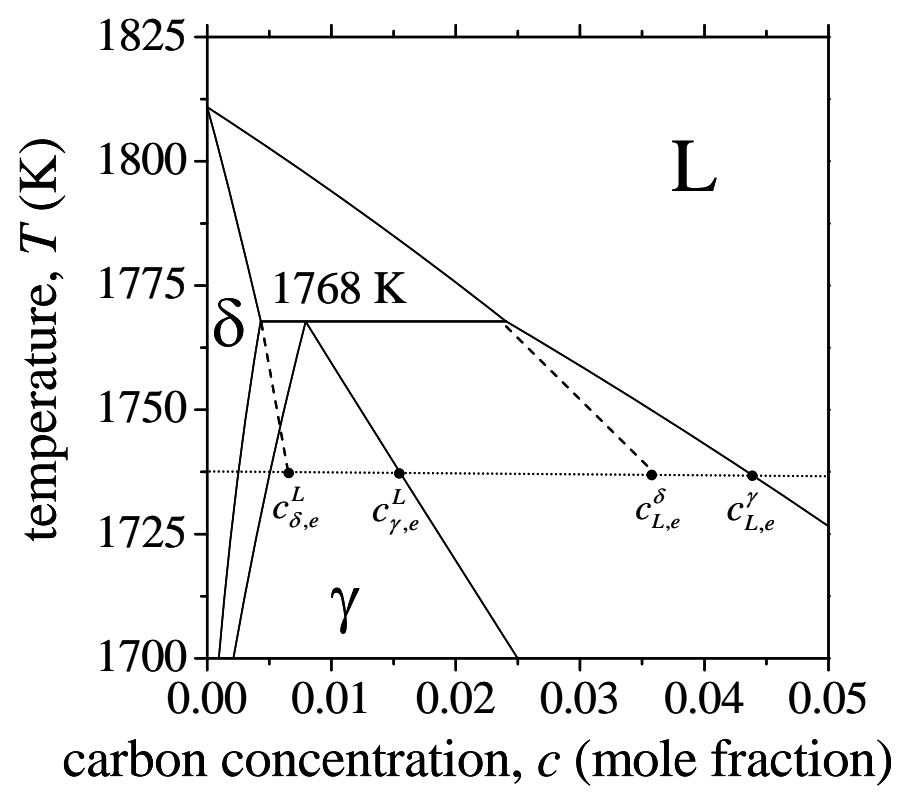

Fig. 1. Phase diagram in Fe-C binary system. 


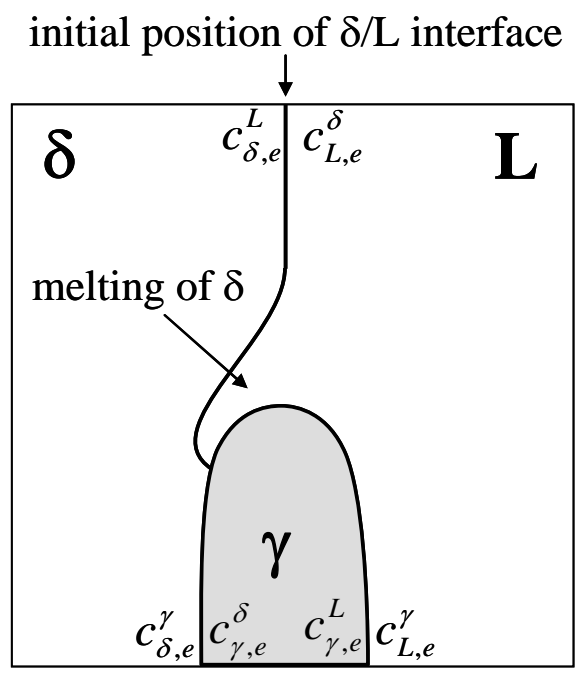

Fig. 2. Shape of interfaces near the triple junction in the peritectic reaction assumed in Ref. [5]. 
(a)

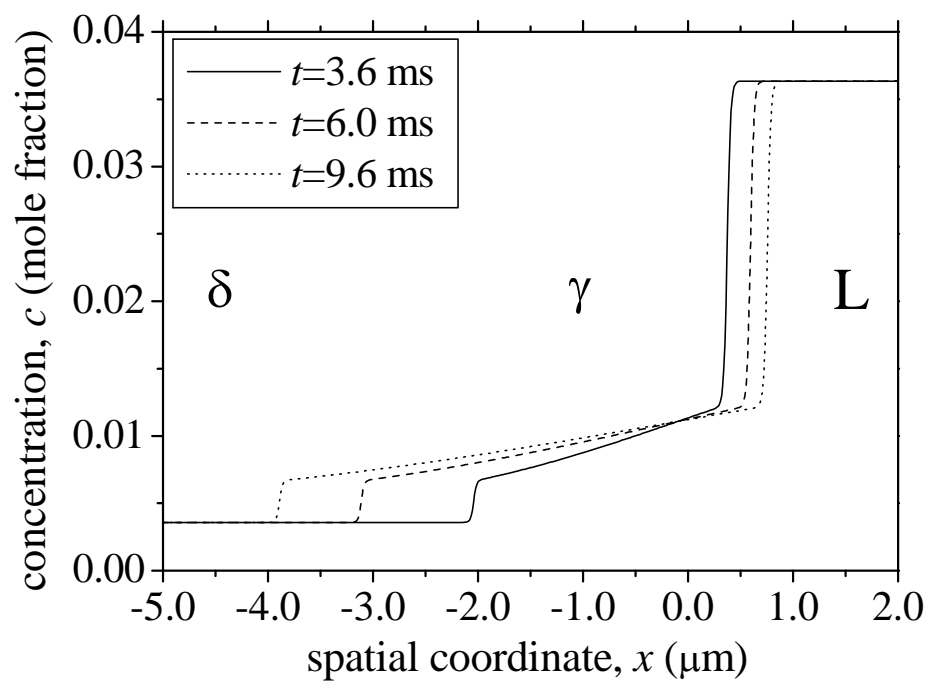

(b)

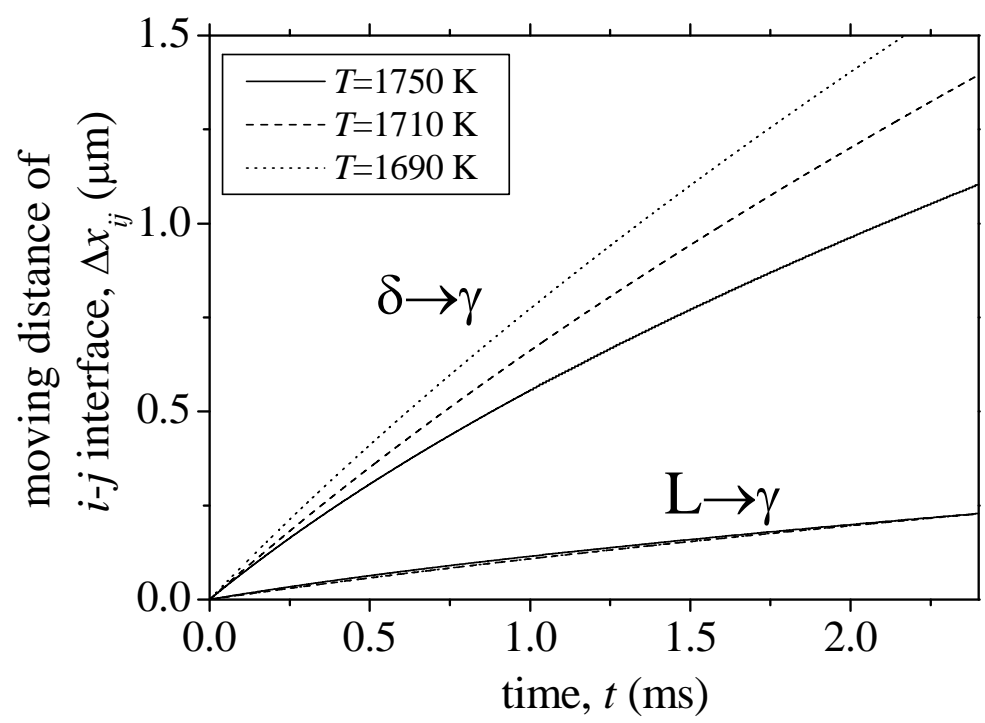

Fig. 3. (a) Concentration profiles at different holding times in isothermal peritectic transformation at $T=1750 \mathrm{~K}$ in one-dimensional system. (b) Time dependence of moving distance of each interface at different temperatures. The results for $\gamma$-L interface at $T=1710$ and $1690 \mathrm{~K}$ are almost superimposed. 


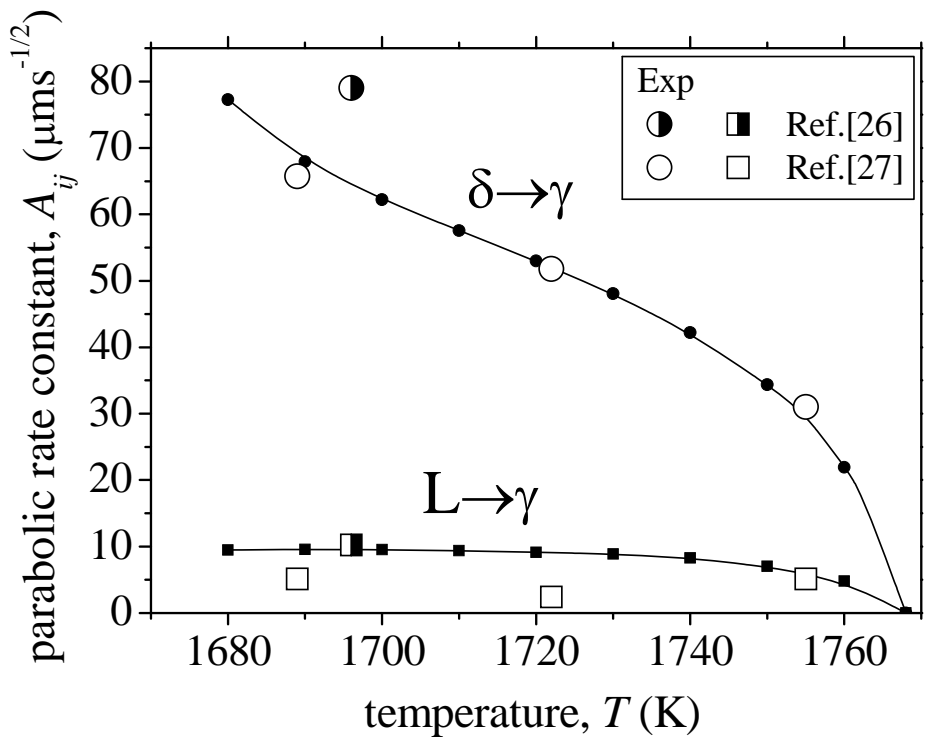

Fig. 4. Dependence of parabolic rate constants for $\delta \rightarrow \gamma$ transformation and $\mathrm{L} \rightarrow \gamma$ solidification on the holding temperature. 


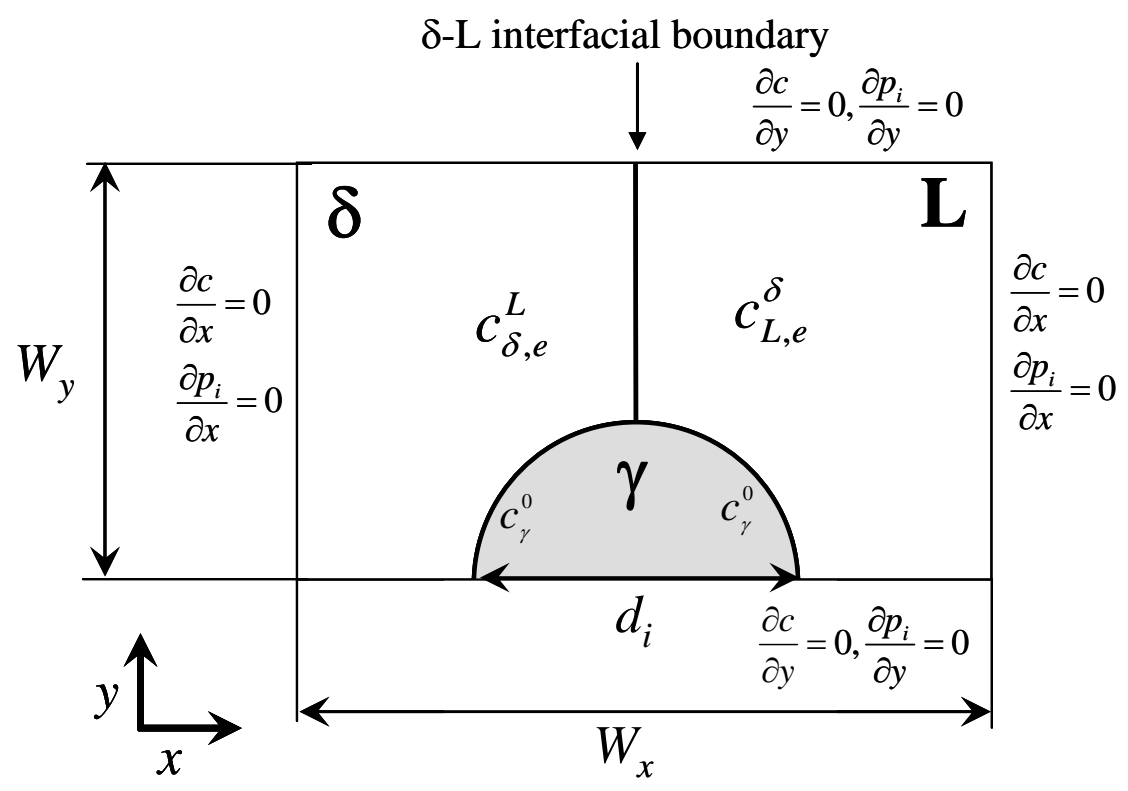

Fig. 5. Schematic illustration of initial state in two-dimensional system for the simulation of the peritectic reaction process. 
(a)

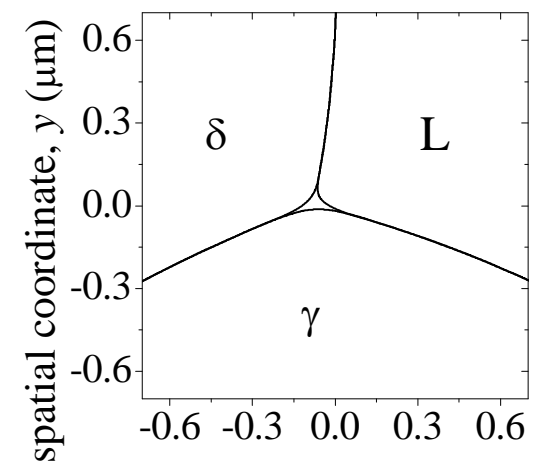

(b)

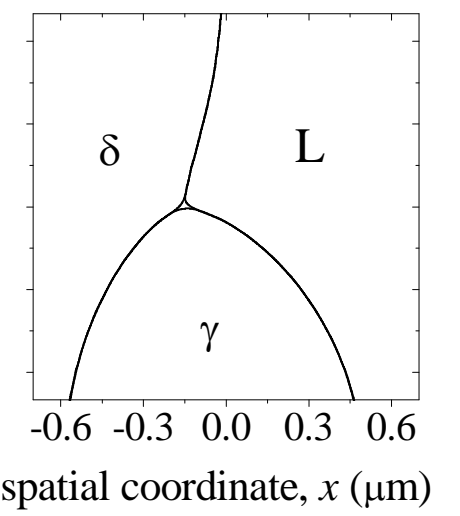

(c)

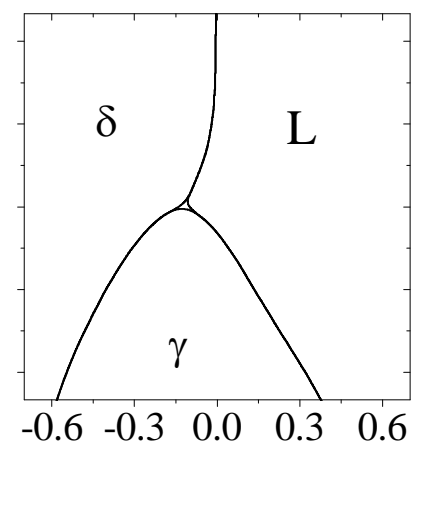

Fig. 6. Shape of interfaces in the vicinity of the triple junction in the peritectic reaction at (a) $\Delta T=0.25 \mathrm{~K}$, (b) $\Delta T=5 \mathrm{~K}$, (c) $\Delta T=10 \mathrm{~K}$. 
(a)

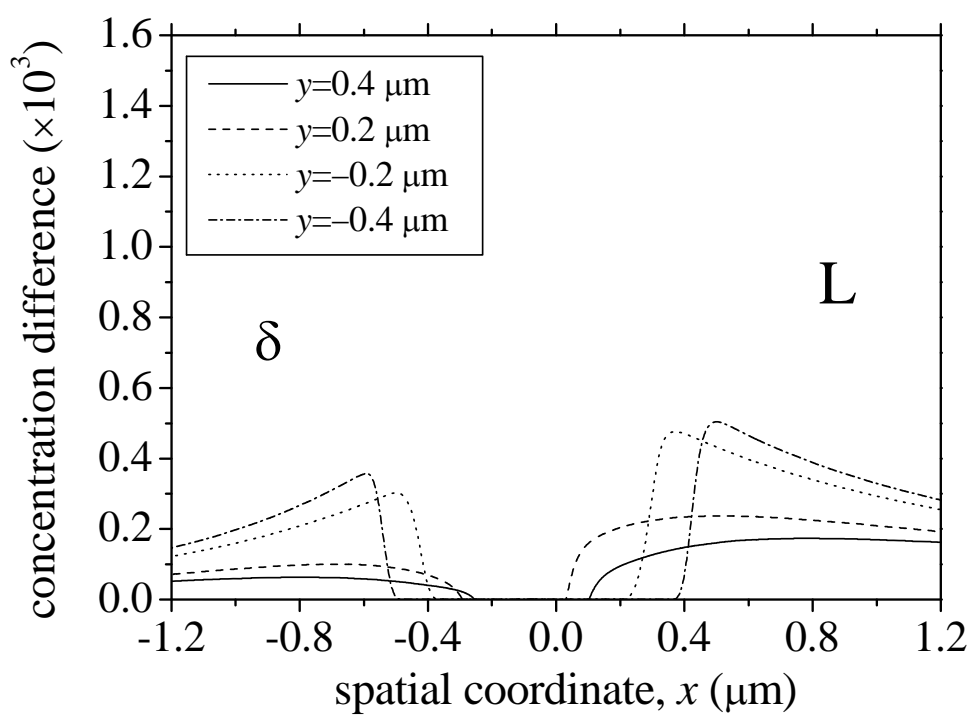

(b)

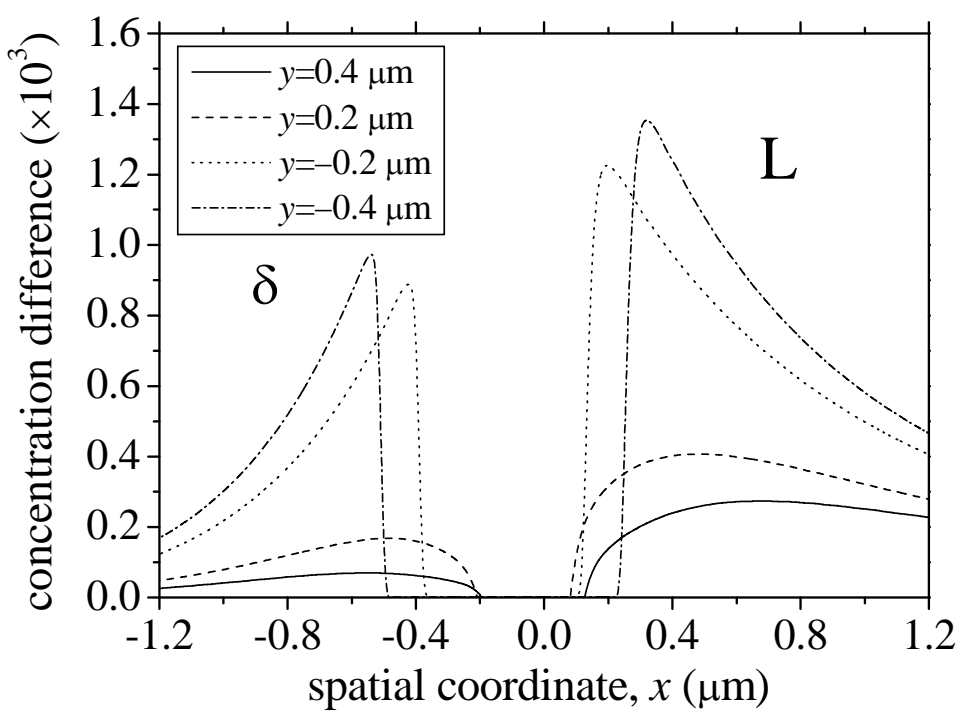

Fig. 7. Concentration profile in $\delta$ phase $\left(c_{\delta, e}^{L}-c\right)$ and $L$ phase $\left(c-c_{L, e}^{\delta}\right)$ near the triple junction at (a) $\Delta T=5 \mathrm{~K}$ and (b) $\Delta T=10 \mathrm{~K}$. 


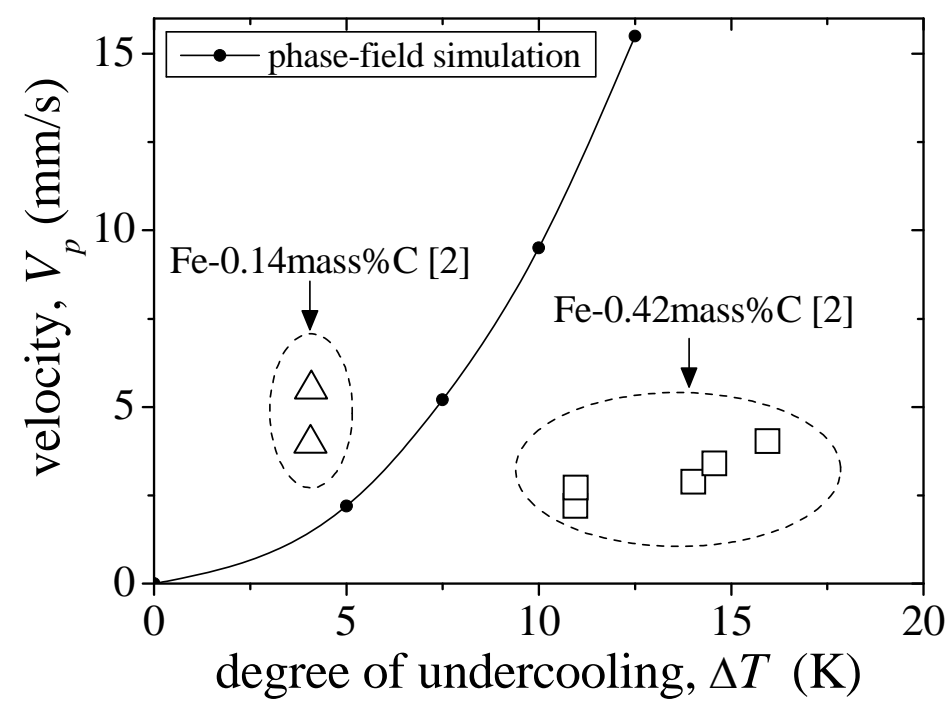

Fig. 8. Dependence of the peritectic reaction rate or the velocity of the front edge of growing $\gamma$ phase on degree of undercooling. 
(a)

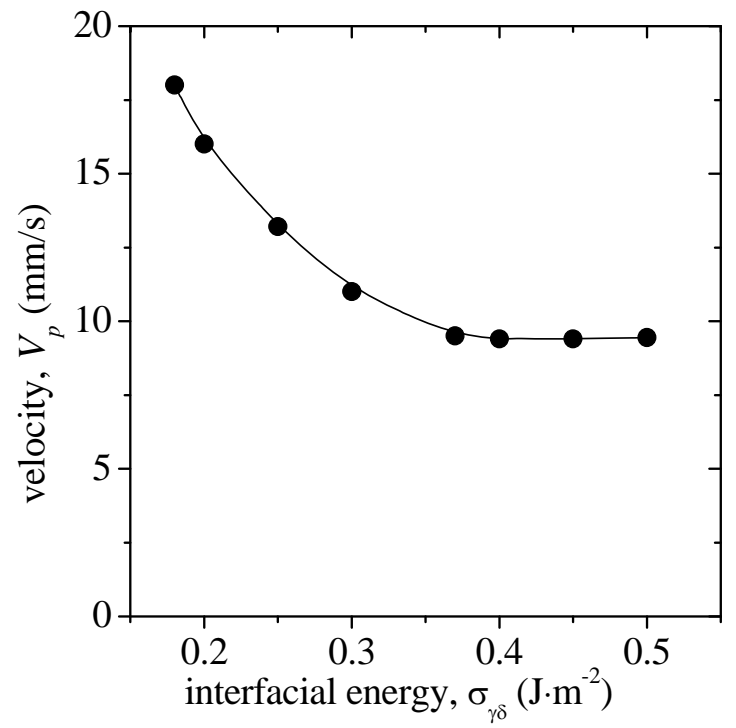

(b)

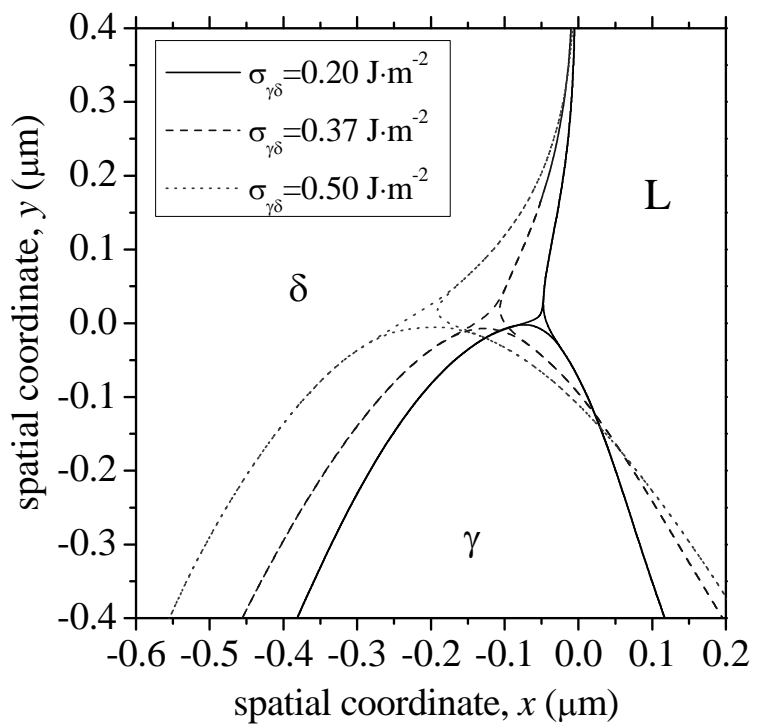

Fig. 9. (a) Dependence of peritectic reaction rate at $\Delta T=10 \mathrm{~K}$ on $\gamma-\delta$ interfacial energy. (b) Shape of interfaces in the vicinity of the triple junction calculated for different values of $\sigma_{\gamma \delta}$. 